\title{
Cyto-Morphological, Biochemical and Radiological Correlation of Various Thyroid Lesions at a Tertiary Care Center
}

\author{
Bharati Bhushan Chittawadagi ${ }^{1 *}$, T.M. Karthikeyan ${ }^{1}$ and M Rajeshwari ${ }^{2}$ \\ ${ }^{1}$ Department of Pathology, Karpagam Faculty of Medical Sciences and Research, Coimbatore, Tamil Nadu, India \\ ${ }^{2}$ Department of community medicine, Karpagam Faculty of Medical Sciences and Research, Coimbatore, Tamil Nadu, India
}

\begin{abstract}
Background: Fine needle aspiration cytology (FNAC) is a cost-effective and time saving diagnostic test used to specifically distinguish between benign and malignant lesions by cytology. Aim: To evaluate the cytomorphological pattern, thyroid hormone profile and radiological findings in early detection of various thyroid diseases. Objective: To correlate the findings of FNAC, thyroid hormone profile and radiological features in diagnosis of various thyroid lesions
\end{abstract}

Methods: 100 patients with thyroid swellings underwent FNAC along with complete thyroid hormone profile\& USG Thyroid.

Result: 93 female and 7 male patients were evaluated. Mean age of patients was 38.62 \pm 14.2 . Ninety-four patients had non-neoplastic and 6 had neoplastic lesions. Colloid goiter was the predominant group among non-neoplastic lesions, followed by Hashimoto's thyroiditis whereas follicular neoplasm was seen more frequently among neoplastic group. Majority of patients (71\%) were in euthyroid state, followed by hypothyroid state and least in hyperthyroid state. In our study $88 \%$ of benign lesions were hyper-echoic and all malignant lesions were hypoechoic. Most of the cystic nodules were benign and showed macro-calcification whereas all malignant lesions showed micro-calcification. Most of the benign lesions showed peripheral hypervascularisation and all malignant lesions showed internal hypervascularisation. There was a statistically positive significant correlation between FNAC findings and radiological features with $\mathrm{P}<0.001$.

Conclusion: FNAC and radiological examination play significant role in an accurate diagnosis of neoplastic and non-neoplastic lesions of thyroid gland. Thyroid profile also plays a very important role in sub-typing of non-neoplastic lesions.

Keywords: Cytomorphologic Correlation, Thyroid FNAC, Thyroid Lesions, USG Thyroid

\section{Introduction}

In general population the incidence of clinically apparent thyroid nodules is $4-5 \%$. ${ }^{[1]}$ Some studies showed an overall prevalence of hypothyroidism to be $5.4 \%$ and hyperthyroidism $1.9 \%$ in goitres. Colloid goitre is the most common thyroid lesion followed by Hashimoto's thyroiditis. ${ }^{[2]}$ Prevalence of thyroid nodules in a given population is affected by a variety of factors such as iodine intake within a given population, age, sex, dietary factors $\&$ radiation exposure.

Incidental finding of a malignancy in the specimens who underwent thyroidectomy for benign disease ranges from $3-16 \%$ of cases. ${ }^{[3]}$ Despite the fact that there is high prevalence of thyroid nodules in general population and higher finding of incidental malignancies in post-operative thyroid specimens, the prevalence of clinically evident thyroid carcinoma is much lower. An accurate diagnosis of thyroid nodules is very important for appropriate clinical management of these patients and to avoid unnecessary surgical interventions.

FNAC of thyroid lesions is a fast, safe, reliable, minimally invasive, cost effective and reaching high sensitivity and specificity and has become a preferred test in the evaluation of thyroid nodules over the past two decades. ${ }^{[4]}$

Introduced in 1967 by Fujimoto et al, ${ }^{[5]}$ thyroid sonography has been widely practiced since 1970 . Now it is one of the most popular radiological methods for diagnosing thyroid lesions. ${ }^{[6]}$. After clinical examination, sonography is commonly the first imaging modality. Ultrasonography is an easily accessible, non-invasive method to image the thyroid gland and its pathology. It helps to detect a possible thyroid abnormality at an early stage and also provides differential diagnosis that result in subsequent thorough examination and timely treatment in appropriate cases.

Hence, this study was undertaken to study the spectrum of different thyroid lesions with clinico-pathological, biochemical and radiological evaluation of non-neoplastic and neoplastic lesions of thyroid gland.

\section{Materials and Methods}

This prospective observational study was conducted in the department of pathology, Karpagam Faculty of Medical Sciences and Research, Coimbatore from March 2015 to March 2017. Ethical clearance was obtained from the 
institutional ethics committee. A total of 100 cases were analyzed during this period. All new patients both male and female in the age group of 15-80 years having thyroid nodules were included in the study. Exclusion criteria: Already diagnosed cases of thyroid lesions \& those patients receiving either thyroxine or any other drug known to interfere with thyroid function at the time of evaluation.

Data Collection: All data relating to the Baseline demographic details such as age, sex, geographic location, clinical characteristics of the patients, thyroid function tests was collected. Radiological examination was done for all 100 patients. The Ultrasound machine used was GE VOLUSON P8 with a $12 \mathrm{MHz}$ linear high frequency probe. A total of 89 thyroidectomy specimens were processed in an automated tissue processor and stained with routine $\mathrm{H}$ and $\mathrm{E}$ stain for histopathological examination.

Local examination of the thyroid is done in all cases to assess the clinical characteristics of the thyroid swelling to note the movement of the thyroid swelling on deglutition. Procedure was done with the patient in supine position with a pillow beneath the shoulder for better visualization of the gland. Using sterile precautions, FNAC was performed using non-aspiration or aspiration technique with the help of $23 \mathrm{G}$ needle attached to $10 \mathrm{ml}$ syringe. The smears were prepared immediately from the aspirates. Majority of the smears were fixed immediately in a fixative containing $100 \%$ ethyl alcohol and stained by hematoxylin and eosin ( $\mathrm{H} \& \mathrm{E})$ stain and examined under light microscope.

Few smears were air dried and stained with Leishman stain and examined under light microscope. In case of cystic swellings, the cyst fluid was collected in the syringe and cyto-centrifuged slides were prepared using Cellspin I.

Collected data from the proforma was entered into Microsoft excel 2016 and analyzed MedCalc software program version 14.8.1. Qualitative variables were described by percentage. A P-value less than 0.05 was considered significant.

\section{Result}

A total of 100 cases of thyroid swellings were analyzed. Demographic variables such as age and sex distribution were explained in Table-1. In this study, total number of females were $93(93 \%)$ and males were $7(7 \%)$. Female to male ratio was $13.2: 1$. The mean age of patients in this study was $38.62 \pm 14.2$ years and age range was $15-80$ years. Age of the youngest patient was 15 years.

In this study, we found that most of the patients were females and most common age group affected was 3140yrs with $28 \%$ (Table-1). All patients clinically presented with neck swelling. Out of 100 patients, 20(20\%) gave history of difficulty in swallowing and 5(5\%) gave history of difficulty in breathing.

Distribution of cases according to site of involvement showed right lobe involvement in 52 cases (52\%), left lobe in 25 cases $(25 \%)$, both lobes involved in 21 cases $(21 \%)$ and isthmus alone in 2 cases $(2 \%)$. In the present study, 94(94\%) patients had benign lesions and 6(6\%) had neoplastic lesions.

Distribution of cases according to cytodiagnosis is explained in Table-2. As seen from table-2, colloid goitre (Figure-1) was the predominant group among nonneoplastic lesions, followed by Hashimoto's thyroiditis (Figure-2), Lymphocytic thyroiditis (Figure-3), Cystic lesions (Figure-4) and de Quervain thyroiditis, whereas follicular neoplasm (Figure-5) was seen more frequently than the papillary carcinoma among neoplastic group.

Distribution of the cases according to hormonal status is explained in Table-3. As seen from Table-3, majority of patients 71/100 (71\%) were in euthyroid state, followed by hypothyroid state and least in hyperthyroid state.

In Non-neoplastic group, more number of patients with colloid goitre have euthyroid state $(74.1 \%) \&$ in case of neoplastic lesions, out of 4 cases of follicular neoplasms; 3 were euthyroid and 1 was hypothyroid. Both cases of papillary carcinoma were euthyroid.

Radiological examination was done for all 100 patients. Out of 100 cases, 88 lesions were hyper-echoic and were benign. 12 lesions (10 benign and 2 malignant) hypo-echoic. Out of 100 cases, 98 lesions had well defined borders and were benign. 2 had ill-defined borders and were malignant. Out of 100 cases, 67 lesions showed macro-calcification and were benign. Two cases showed typical micro-calcification and were malignant and 31 benign lesions did not show calcification.

Out of 100 cases, 74 lesions showed peripheral hypervascularisation and were benign. 10( 8 benign and 2 malignant) showed internal (central) hypervascularisation and 16 lesions with absent flow were benign. On ultrasonography most of the benign lesions showed cystic degeneration. There was a statistically positive significant correlation between FNAC findings and radiological features for different thyroid lesions with $\mathrm{P}<0.001$.

\section{Discussion}

Thyroid nodules occur with a prevalence of $4 \%$ \& majority occurring in the age group between 30 and $60 .{ }^{[7]}$ Most of the swellings are benign and only $10-20 \%$ are malignant. ${ }^{[7]}$ So surgery as the initial intervention will have a very low yield. Despite remarkable technical advancement 
Table 1: Age wise distribution of Cases $(n=100)$.

\begin{tabular}{|c|c|c|c|c|}
\hline Sr.no & Age & Male, $\mathbf{n}(\%)$ & Female, $\mathbf{n ~ ( \% ) ~}$ & Total, $\mathbf{n}(\%)$ \\
\hline 1 & $11-20$ & 0 & $13(13 \%)$ & $13(13 \%)$ \\
\hline 2 & $21-30$ & 0 & $19(19 \%)$ & $19(19 \%)$ \\
\hline 3 & $31-40$ & $2(2 \%)$ & $26(26 \%)$ & $28(28 \%)$ \\
\hline 4 & $41-50$ & $1(1 \%)$ & $18(18 \%)$ & $19(19 \%)$ \\
\hline 5 & $51-60$ & $3(3 \%)$ & $13(13 \%)$ & $16(16 \%)$ \\
\hline 6 & $61-70$ & $1(1 \%)$ & $3(3 \%)$ & $4(4 \%)$ \\
\hline 7 & $71-80$ & 0 & $1(1 \%)$ & $1(1 \%)$ \\
\hline 8 & Total & $\mathbf{7 ( 7 \% )}$ & $\mathbf{9 3 ( 9 3 \% )}$ & $\mathbf{1 0 0 ( 1 0 0 \% )}$ \\
\hline
\end{tabular}

Table 2: Distribution of cases according to cytodiagnosis, $\mathbf{n = 1 0 0 .}$

\begin{tabular}{|c|c|c|c|c|}
\hline SI.No & Non-neoplastic & No. of cases, $n(\%)$ & Neoplastic & No. of cases, $n(\%)$ \\
\hline 1 & Colloid Goitre & $53(53 \%)$ & Follicular neoplasm & $4(4 \%)$ \\
\hline 2 & Cystic lesions & $2(2 \%)$ & Papillary carcinoma & $2(2 \%)$ \\
\hline 3 & Hashimoto's thyroiditis & $26(26 \%)$ & & \\
\hline 4 & Lymphocytic thyroiditis & $12(12 \%)$ & & \\
\hline 5 & de Quervain thyroiditis & $1(1 \%)$ & & \\
\hline
\end{tabular}

Table 3: Distribution of cases according to hormonal status, $\mathbf{n = 1 0 0 .}$

\begin{tabular}{|c|c|c|c|c|}
\hline Sr.no & Cytodiagnosis & Euthyroid, $\mathbf{n}$ & Hyperthyroid, $\mathbf{n}$ & Hypothyroid, $\mathbf{n}$ \\
\hline 1 & Goitre & 40 & 6 & 7 \\
\hline 2 & Cystic lesions & 2 & 3 & 6 \\
\hline 3 & Hashimoto's thyroiditis & 17 & 2 & 4 \\
\hline 4 & Lymphocytic thyroiditis & 6 & - & - \\
\hline 5 & de Quervain thyroiditis & 1 & - & 1 \\
\hline 6 & Follicular neoplasm & 3 & - & - \\
\hline 7 & Papillary carcinoma & 2 & $\mathbf{1 1}$ & $\mathbf{1 8}$ \\
\hline $\mathbf{8}$ & Total & $\mathbf{7 1}$ & & \\
\hline
\end{tabular}

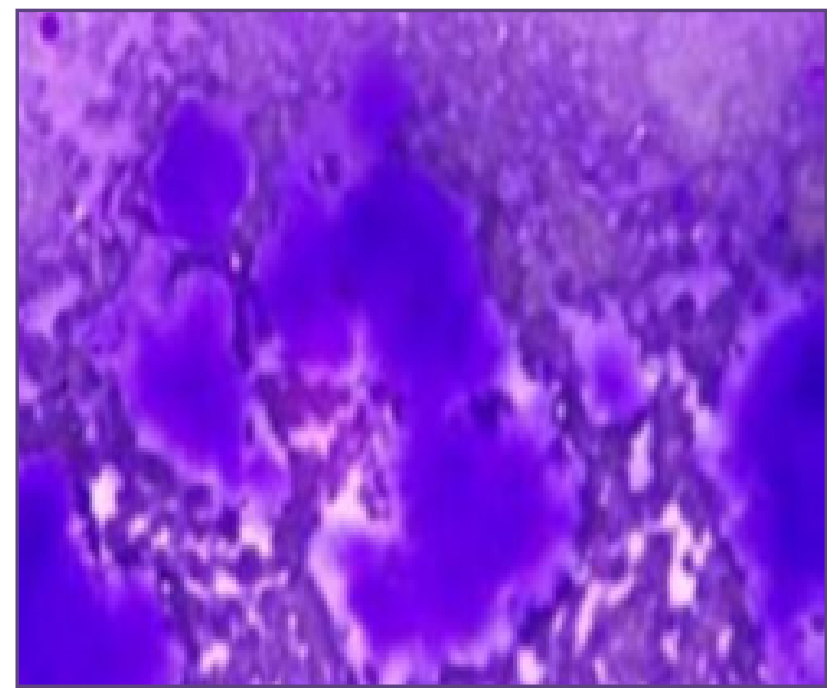

Fig. 1: Thick Colloid-Colloid Goitre. Leishman stain [10X].

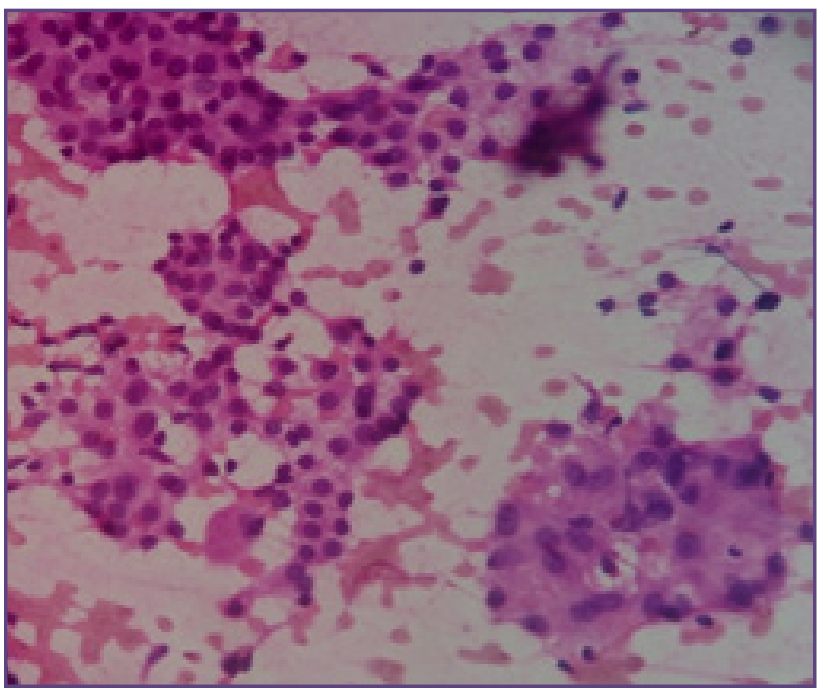

Fig. 2: Hashimotos Thyroiditis. H\&E stain [40X]. 


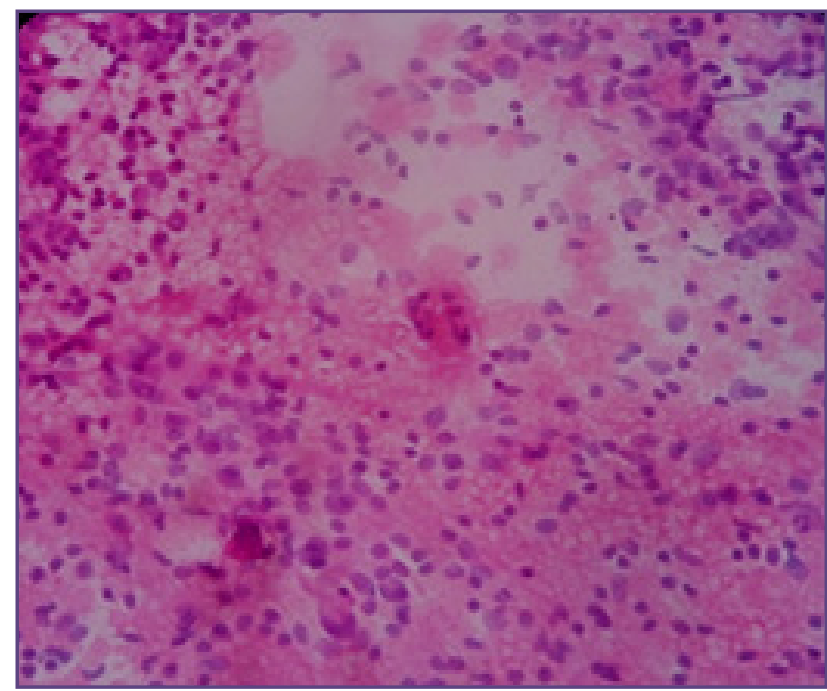

Fig. 3: Lymphocytic thyroiditis. H\&E stain [40X].

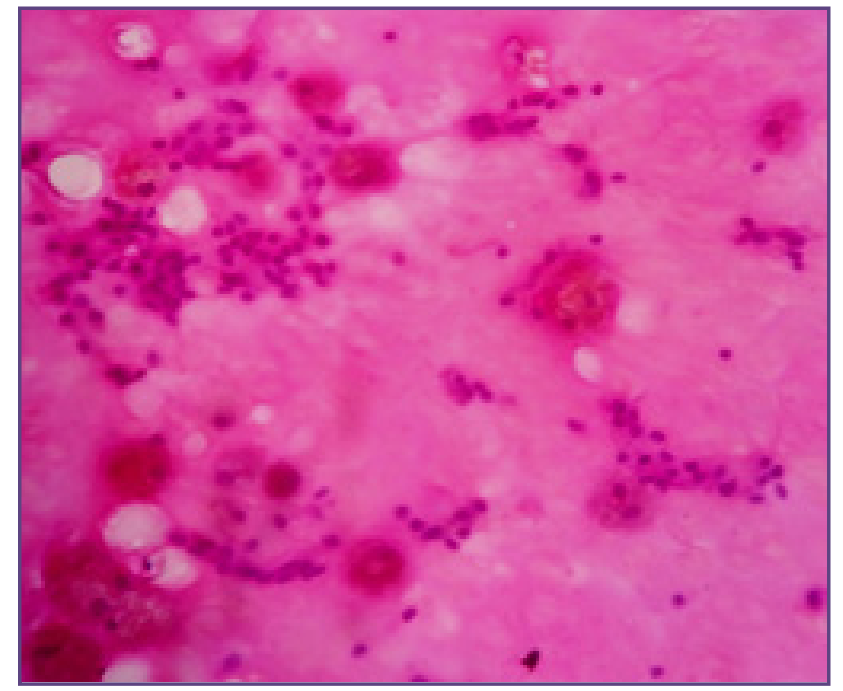

Fig. 4: Colloid cyst. H\&E stain [40X].

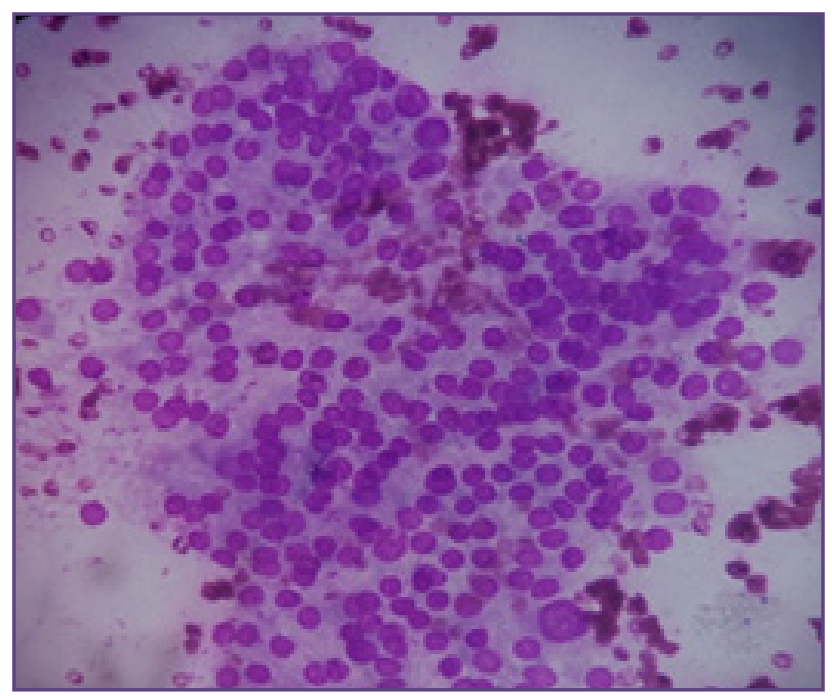

Fig. 4: Colloid cyst. H\&E stain [40X].

in diagnostic procedure over the last decade, there is potential for misdiagnosis. Very few diagnostic tests are useful to differentiate benign lesions from malignant. Thyroid ultrasound can be used to distinguish solid lesions from cystic lesions. But all cystic lesions are not benign. Due to excellent visualization of the thyroid parenchyma, high resolution ultrasound has become a first line imaging modality for evaluation of the thyroid gland. Ultrasonography is highly sensitive in detecting small nodules, calcification, septations, type of borders and cystic change and also helps in guiding fine needle aspiration cytology. ${ }^{[8]}$

Fine needle aspiration cytology is usually the first line of investigation for any kind of thyroid lesion and the other investigations are done subsequently to select the patients who require surgery and those that can be managed conservatively.

In our study, females are the predominant group affected by thyroid lesions and the most common age group affected by thyroid lesion was in $3^{\text {rd }}$ decade, with a comparable data support from literature review as seen in a study by Gupta et al. ${ }^{[9]}$

In the present study, most of the thyroid lesions were non-neoplastic (94\%) with neoplastic thyroid lesions constituting only $6 \%$ of the cases which is comparable with a study done by $\mathrm{S}$ Chandanwale et al. ${ }^{[10]}$

Among non-neoplastic lesions, colloid goitre formed the predominant group (53\%) and was in accordance with a study by $\mathrm{S}$ Chandanwale et al and we found that cyto- 
histological concordance shows a statistically significant positive correlation $(\mathrm{P}<0.05)$ for non-neoplastic lesion which were close to the studies done by Gupta et al, ${ }^{[9]}$ and Basharat et al. ${ }^{[1]}$

In the present study the most common neoplastic lesion was follicular neoplasm, however in studies done by $\mathrm{S}$ Chandanwale et al ${ }^{[10]}$ and Tabaqchali et al, ${ }^{[12]}$ the most common neoplastic lesion was papillary carcinoma of thyroid.

In our study, in non-neoplastic group, more number of patients with colloid goitre had euthyroid state $(74.1 \%) \&$ in case of neoplastic lesions, out of 4 cases of follicular neoplasms; 3 were euthyroid and 1 was hypothyroid. Both cases of papillary carcinoma were euthyroid. In a study by Chaudhary et al, ${ }^{[7]}$ most patients $(61.3 \%)$ had euthyroid status in both in neoplastic and non-neoplastic group.

In our study $88 \%$ of benign lesions were hyper-echoic and both malignant lesions were hypo-echoic which was in comparison with the statement made by Brkljacic et al ${ }^{[13]}$ that hypoechogenicity is associated with thyroid malignancy. These results are in agreement with other literatures, that most of the benign nodules are hyper-echoic and most of the malignant nodules are hypo-echoic. ${ }^{[1,15]}$

Our study revealed that most of the cystic nodules were benign and showed macro-calcification whereas all malignant lesions showed micro-calcification which was in accordance with other studies. ${ }^{[15,16]}$

In our study most of benign lesions showed well defined borders and all malignant lesions showed ill-defined borders which was comparable to the study done by Amer AM Ali et al, ${ }^{[17]}$ that $80 \%$ of malignant nodules had irregular borders. Most of the benign lesions showed peripheral hypervascularisation and all malignant lesions showed internal hypervascularisation which was similar to the study done by Laith A Khalaf, ${ }^{[18]}$ study found that internal hypervascularisation was seen in $62.5 \%$ of malignant lesions. And we found that there is a statistically significant positive correlation between FNAC findings and radiological features for different thyroid lesions with $\mathrm{P}<0.001$.

\section{Conclusion}

From our present study, we conclude that FNAC \& Ultrasound helps to identify thyroid abnormalities at an early stage. Ultrasound \& FNAC gives us strong positive statistically significant correlation in diagnosing various benign and malignant lesions of thyroid gland. Biochemical analysis also gives us additional supporting evidence.

\section{Reference}

1. Gharib H, Papini E, Valcavi R, et al. American Association of Clinical Endocrinologists and Associazione Medici Endocrinologi medical guidelines for clinical practice for the diagnosis and management of thyroid nodules. EndocrPract 2006; 12:63-102.

2. Gayathri BN, Kalyani R, Harendra Kumar ML, Krishna Prasad K. Fine needle aspiration cytology of Hashimoto's thyroiditis - a diagnostic pitfall with review of literature. Journal of Cytology. 2011;28(4):210-213.

3. Miccoli P, Minuto MN, Galleri D et al. Incidental thyroid carcinoma in a large series of consecutive patients operated on for benign thyroid disease. ANZ J Surg2006; 76: 123-6.

4. Handa U, Garg S, Mohan H, Nagarkar N. Role of the fine needle aspiration cytology in diagnosis and management of thyroid lesions: A study on 434 patients. J Cytol 2008; 25:13-7.

5. Fujimoto F, Oka A, Omoto R, Hirsoe M. Ultrasound scanning of the thyroid gland as a new diagnostic approach. Ultrasonics, 1967; 5: 177-80.

6. Bruno A., Policeni Wendy, R.K Smoker, Deborah L. Reede. Anatomy and Embryology of the thyroid and parathyroid glands. Semin ultrasound CT MRI, 2012; 33: 104-114.

7. Ritica Chaudhary, Zulfikar Ahmed, Umaru N. A Correlative study of FNAC Thyroid with Thyroid Hormone Profile. Journal of Evolution of Medical and Dental Science 2014; Vol.3 Issue 06, February 10; Page :1474-1480.

8. Mandrekar SR, Nadkarni NS, Pinto RG, Menezes S. Role of fine needle aspiration as the initial modality in investigation of thyroid lesions. ActaCytol 1995; 39:898-903.

9. Monoj Gupta, Savita Gupta, and Ved Bhushan Gupta. Correlation of Fine Needle aspiration Cytology with Histopathology in the Diagnosis of Solitary Thyroid Nodule. Journal of Thyroid Research Volume 2010, Article ID 379051, 5 pages 2010 .

10. Chandanwale S, Singh N, Kumar H, Pradhan P, Gore C, Rajpal M. Clinicopathological correlation of thyroid nodules. Int J Pharm Biomed Sci 2012; 3:97-102.

11. Basharat R, Bukhari MH, Saeed S, Hamid T. Comparison of fine needle aspiration cytology and thyroid scan in solitary thyroid nodule. Patholog Res Int 2011; 2011:754041.

12. Tabaqchali MA, Hanson JM, Johnson SJ, Wadehra V, Lennard TW and Proud G. Thyroid aspiration cytology in Newcastle. Am R Coll Surg Engl 2000; 82: 149-155.

13. Brkljacic B, Cuk V, Tomic-Brzac H, et al. Ultrasonic evaluation of benign and malignant nodules in echographically multinodular thyroids. J Clin Ultrasound. 1994; 22:71-76.

14. Belfiore A, La Rosa GL, La Porta GA. Cancer risk in patients with cold thyroid nodules: relevance of iodine intake, sex, age, and multinodularity. Am J Med. 1992; 93:363-9.

15. Nayar R, Nemcek A. Radiologic and pathologic features of thyroid calcifications: A viewpoint. Pathology case review. 2003; 8:22-24. 
16. Bryan K, Terry S, Ross M, Ronald J, Brooke J. Common and uncommon sonography features of papillary carcinoma. J ultrasound med2003; 22:1083-1090.

17. Amer AM Ali, Abdulkader H Hasan, Tahir A Hawrami. The role of conventional ultrasound in the assessement of the thyroid nodules in Al-Sulaimanyia territory. Bas J Surg. 2009;15:34.

18. Laith A Khalaf, Hussein A Abdul-Shaheed. The value of ultrasound and color- Doppler features in the assessment of single solid thyroid nodule. Iraqi JMS. 2012; 10:287-292.

*Corresponding author:

Dr. Bharati Bhushan Chittawadagi, No D 14,block 2,patteshwarar apt, Opp to gem hospital, ramanathapuram, Coimbatore, India,Tamil nadu 641045

Phone: +91 8940770993

Email: docbharati@gmail.com

Financial or other Competing Interests: None. 